\title{
Correlation of hepatic fibrosis assessed by Metavir score and digital morphometry in a murine model
}

\author{
Nathalie Alemán-García1, Celia Sánchez-Pérez ${ }^{1 *}$, Adolfo Pérez-García ${ }^{2,3}$, Marco A. Durán-Padilla \\ David Kershenobich ${ }^{5}$, and Joselín Hernández-Ruíz ${ }^{3,6}$
}

${ }^{1}$ Applied Sciences and Technology Institute, National Autonomous University of Mexico, Mexico City, Mexico; ${ }^{2}$ Experimental Surgery Service, Hospital General de México "Dr. Eduardo Liceaga", Mexico City, Mexico; ${ }^{3}$ Experimental Medicine Unite, School of Medicine, National Autonomous University of Mexico, Mexico City, Mexico; ${ }^{4}$ Patology Service, Hospital General de México "Dr. Eduardo Liceaga", Mexico City, Mexico; ${ }^{5}$ Instituto Nacional de Ciencias Médicas y Nutición Salvador Zubirán, Mexico City, Mexico; ${ }^{6}$ South Texas Diabetes and Obesity Institute, University of Texas Rio Grande Edinburg, Texas, United States of America

\begin{abstract}
Background: Animal models for hepatic fibrosis are currently used for clinical studies, and research works aimed to the progression and regression of the disease. In these, fibrosis is evaluated using histopathological assessment with high variability inter- and intra-observers. Digital morphometry in the space color $L A B$ could reduce this variability; however, its correlation degree with the histopathological assessment is unknown. Objective: The objective of the study was to analyze the correlation between digital morphometry and the histopathological assessment of hepatic fibrosis in images of biopsies of a murine model. Materials and methods: We performed a descriptive, experimental, longitudinal, prospective, analytical, and correlational study. Fibrosis was induced by intraperitoneal injection of carbon tetrachloride in 16 Wistar male rats. We did a postmortem hepatectomy and took histological specimens stained by Masson trichrome, obtaining ten randomly selected images at $\times 10$ in each liver. We quantified the fibrosis percentage (FP) by digital morphometry and compared it to the Metavir score given by three independent evaluators. FP was analyzed by a ANOVA test and Spearman Rho test. Results: The evaluated images had 70\% concordance of the three evaluators. The analysis of the FP revealed fibrosis evaluation groups with statistical differences. The FP had a very high correlation with the histopathological diagnosis $(p=0.0001, r=0-9)$. The ranges obtained from FP for each phase of fibrosis were: $0.4 \%$ for $F 0,0.44-1.14 \%$ for $F 1,1.52-2.74 \%$ for F2, 3.1-3.8\% for F3y, and 5.12-6.12\% for F4. Conclusions: The morphometric quantification had a high correlation with the histopathological evaluation of images of histological sections of liver biopsies of the murine model of fibrosis. The quantification of the FP by digital morphometry will be useful to increase the accuracy in the evaluation of liver fibrosis.
\end{abstract}

Key words: Hepatic fibrosis. Murine model. Metavir score. Digital morphometry.

\section{Introduction}

Fibrosis is described as the biological evidence of an error in the dynamic process of cellular repair ${ }^{1}$. Liver cirrhosis is the final pathological condition resulting from persistent damage in the liver, in which healthy tissue is replaced by scar tissue, which causes a decompensation and alteration in its functioning and architecture, often irreversible. Cirrhosis represents an important cause of morbidity and mortality in the world population ${ }^{2}$. In some countries of Latin America, it occupies between the fifth and sixth place as a cause of general death. In Mexico, the mortality due to liver cirrhosis in 2013 was positioned as cause number five ${ }^{3}$. Although this condition is considered a problem that
Correspondence:

${ }^{\star}$ Celia Sánchez-Pérez

E-mail: celia.sanchez@ icat.unam.mx

Available online: 17-01-2020

Date of reception: 10-08-2018

Date of acceptance: 25-09-2018 DOI: 10.24875/HGMX.M20000034
Rev Med Hosp Gen Mex. 2020;83(1):13-19 www.hospitalgeneral.mx 0185-1063/@ 2018 Sociedad Médica del Hospital General de Mexico. Published by Permanyer. This is an open access article under the CC BYNC-ND license (http://creativecommons.org/licenses/by-nc-nd/4.0/). 
implies the loss of global health, there is not enough, accurate, and updated information on the epidemiological scope and, moreover, on the precise clinical characteristics of its evolution and reversion processes, thus limiting the possibilities of more adequate prevention, intervention, and assistance ${ }^{4}$.

To contribute to the study of this pathology, multiple trials have been conducted using animal models. One of the methods frequently found in the literature, due to its simplicity and relative reproducibility, is the use of substances that generate a significant activation of the hepatic stellar cells, which causes an increase in the production of extracellular matrix, inflammation, and necrosis ${ }^{5}$. Some of the components used for these models are, for example, dimethylnitrosamine, galactosamine, and carbon tetrachloride $\left(\mathrm{CCl}_{4}\right)$. An important limitation in the study of liver cirrhosis is the subjective evaluation of the progression of fibrosis, which in experimental trials is commonly estimated from the quantification of serum markers and anatomopathological studies of the biopsy ${ }^{6-8}$. In the latter case, a pathology specialist performs qualitative assessments of the structural characteristics of the tissue. The inter- and intra-observer variability of the diagnoses, once the biopsy is taken, is also criticized and represents an important factor of variability ${ }^{9}$. Although there are different classification systems for liver fibrosis, one of the most frequently used is the Metavir score ${ }^{10}$.

Several works have been aimed to the development of digital image analysis methods for the quantification of hepatic fibrosis ${ }^{11-15}$. Dahab et al. ${ }^{11}$. developed an automated method for the digital quantification of fibrosis in liver images of rats making correlation studies on a qualitative conventional scale. The automation used is a restriction, since the experience of a skilled observer is disregarded and the evaluations depend mostly on the quality and staining of the histological specimen. En Goodman et al. ${ }^{12}$ presented a study to evaluate an anti-fibrosis agent using a commercial automated system for the acquisition of biopsy images, with a processing in color RGB and grayscales using cross-polarized filters. As a consequence of tissue fragmentation and insufficient length and/or width, 144 cases of the 389 patients had one or both biopsies discarded for analysis. They reported a correlation coefficient of 0.49 between phases of fibrosis in the Ishak score. Although results indicate a degree of average correlation, they showed the importance of applying this type of techniques to be used in future therapeutic trials of agents to inhibit the progression of fibrosis.
Zaitoun et al. ${ }^{13}$ present the quantification of periportal, pericellular, and septal fibrosis; as well as steatosis, by stereological and morphometric techniques, in biopsies of patients with chronic hepatitis $C$ and patients with alcoholic liver disease (ALD). They found a relationship between the quantification of fibrosis by means of the commercial system Profit 5.2 $2^{\mathrm{TM}}$, and the modified histological score of Ishak. However, in patients with cirrhosis, the amount of fibrosis varied between $20 \%$ and $74 \%$. Significant differences were found in the amount of fibrosis between patients with chronic hepatitis $C$ and those with ALD.

In this work, we present a methodology for the quantification of fibrosis in liver images based on morphometric processing of the color represented in the LAB color space of the Commission International de l'Eclairage. In the LAB space, it is possible to represent more colors with respect to the RGB scale; in addition to, better emulate the non-linear response of the human eye. The color representation is given in a three-dimensional space of the components of luminosity (L), transition of red-green $(A)$, and transition of yellow-cyan (B). In this space, the color of the fibrosis selected in the image is represented as a point in the three-dimensional space $L^{*} A * B$. Thus, the final obtaining of the color of the fibrosis is calculated from the selection of three zones identified as fibrosis, where each of the LAB components is averaged to find a resulting color, which is used as the selection value of each pixel of the image in which color matches. The percentage of fibrosis is calculated by counting the pixels that match the selected value with respect to the total number of pixels in the image. We present the correlation between the fibrosis quantification in a murine model and the histopathological evaluation, hence validating the principle of the proposed methodology.

\section{Materials and methods}

We conducted a descriptive, longitudinal, prospective, experimental, analytical, and correlational study.

\section{Animal model of induction of fibrosis by $\mathrm{CCl}_{4}$}

Fibrosis was induced in male Wistar rats of 6 weeks of age, weighing between 190 and $220 \mathrm{~g}$. Four groups of four rats were randomly formed, in which $\mathrm{CCl}_{4}$ was injected intraperitoneally in olive oil, in an initial dose of $250 \mu \mathrm{l}$, at a concentration of $33 \%$, twice a week ${ }^{16,17}$. 
Table 1. Scheduled times in the murine model for each fibrosis induction group

\begin{tabular}{|l|l|}
\hline Group & Time \\
\hline T1 & 4 weeks \\
\hline T2 & 6 weeks \\
\hline T3 & 10 weeks \\
\hline T4 & 13 weeks \\
\hline
\end{tabular}

Table 2. Metavir score for fibrosis evaluation

\begin{tabular}{|l|l|}
\hline Phase & Anatomopathological characteristics \\
\hline F0 & No fibrosis \\
\hline F1 & Portal fibrosis without septa \\
\hline F2 & Portal fibrosis with few septa \\
\hline F3 & Numerous septa. Incomplete nodules \\
\hline F4 & Complete nodules: cirrhosis \\
\hline
\end{tabular}

After individuals gained $20 \%$ of their initial weight, the induction dose was increased weekly to $1 \mu \mathrm{l} / \mathrm{g}$. At the end of the induction time (Table 1), individuals were sacrificed with an overdose of sodium pentobarbital (Pisabental, Pisa agropecuaria ${ }^{\circledR}$ ) intraperitoneally and a total hepatectomy performed.

\section{Liver samples and imaging}

A cut was systematically taken from the right hepatic medial lobe of all the hepatectomized livers and stained with Masson trichrome. Using an optical microscope, the slides were photographed at a $\times 10$, to obtain ten images of each one, controlling that the field of vision completely corresponded to tissue.

\section{Histopathological evaluation of the images in Metavir score}

Three histopathological evaluations were carried out in a blinded and independent manner of each image using the Metavir score, described in table 2. The final evaluation was taken as the most concordant, thus resulting in evaluations with complete agreement, namely $3 / 3$; or evaluations with one non-concordant evaluator, namely, two-third. When the disparity was complete (none of the three evaluators agreed on the evaluation), the opinion of a fourth was requested to perform the conclusive evaluation based on 2/4 coincidences.

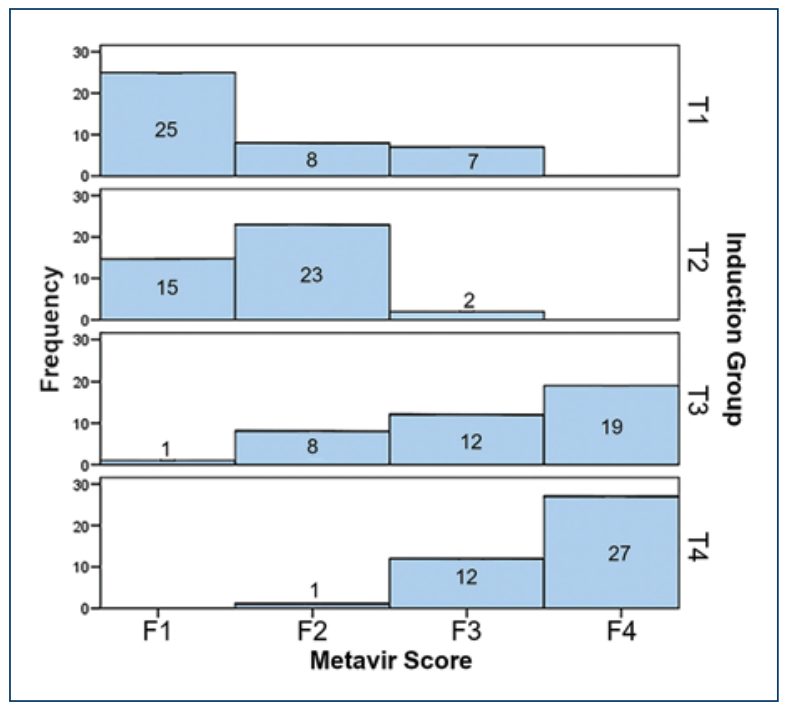

Figure 1. Fibrosis evaluation for the 160 images of the murine model according to the induction groups (T1-T4).

\section{Morphometric quantification of fibrosis}

The quantification of fibrosis was performed in the 160 images taken from the liver tissue. The quantification was performed by the same experienced user, trained in the visual identification of fibrosis and its histological characteristics. The quantification was performed by selecting fibrous tissue from three different areas of the image as distant as possible, to be more representative of possible color differences in the staining.

\section{Statistical analysis}

The concordance between the evaluators for each staging was calculated. The data obtained from the quantification of the fibrosis percentage (FP) were expressed as mean and standard deviation (SD) and to determine statistical differences between the induction groups, an ANOVA test was applied; the significance was determined when $p<0.05$. A Spearman's rho test was used to establish the correlation between the FP variable and the histopathological evaluation. The statistical analysis was carried out with the program SPSS $^{\text {TM }}$, v. 15.

\section{Results}

As previously described, a histological cut stained with Masson trichrome was obtained from each of the 16 individuals and photographed in ten different areas, thus acquiring a total of 160 images. 
Table 3. Analysis of the percentage of fibrosis according to the Metavir evaluation groups

\begin{tabular}{|l|c|c|c|c|}
\hline Metavir score & $\overline{P F_{M}}(\%)$ & $D E_{P F_{M}(\%)}$ & Minimum (\%) & Maximum (\%) \\
\hline F1 & 0.8 & 0.3 & 0.2 & 1.7 \\
\hline F2 & 2.1 & 0.6 & 1.0 & 3.5 \\
\hline F3 & 4.1 & 1.0 & 2.3 & 6.7 \\
\hline F4 & 4.9 & 1.1 & 3.1 & 7.7 \\
\hline
\end{tabular}
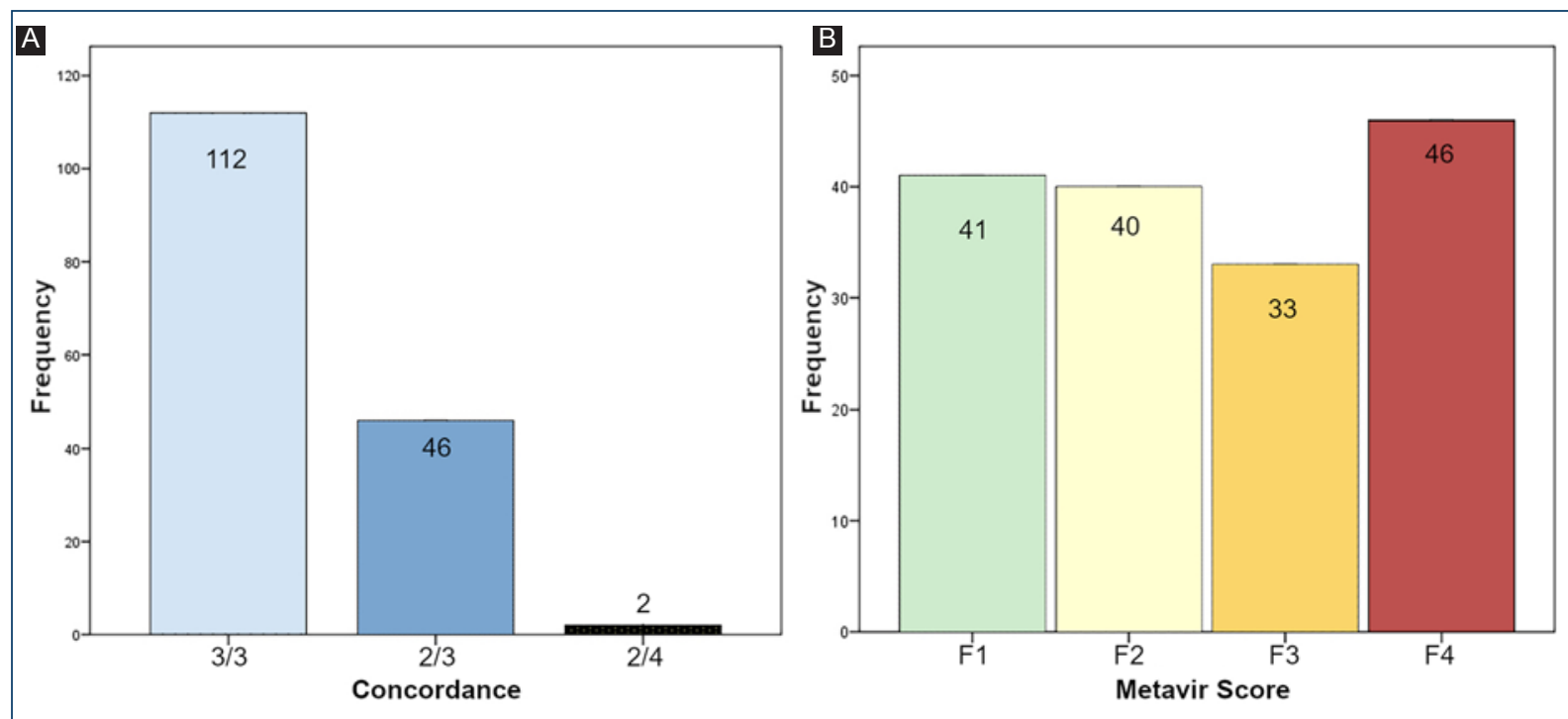

Figure 2. Histological evaluation analysis. A: evaluators coincidence. B: fibrosis evaluation.

\section{Histopathological evaluation}

The range of distribution of fibrosis evaluation in each induction group (T1-T4) is shown in figure 1. The Metavir score evaluation for the 40 images for each induction group overlaps with other fibrosis phases that did not correspond to the expected phase according to the induction time. The concordance in the histopathological evaluation of fibrosis is illustrated in figure $2 \mathrm{~A}$. The frequency of the evaluations of the 160 analyzed images is shown in figure $2 \mathrm{~B}$ according to the Metavir evaluation groups being: 46 in F4, 33 in F3, 40 in F2, 41 in $\mathrm{F} 1$, and no cases in F0.

\section{Quantification of fibrosis}

Using the morphometric algorithm, the FP in the 160 images was calculated. The FP was grouped according to the evaluated Metavir phase, the box plot is shown in figure 3 . Table 3 shows the descriptive statistics for the FP for each group according to the Metavir evaluation. The results of the ANOVA test show significant statistical differences for the Metavir

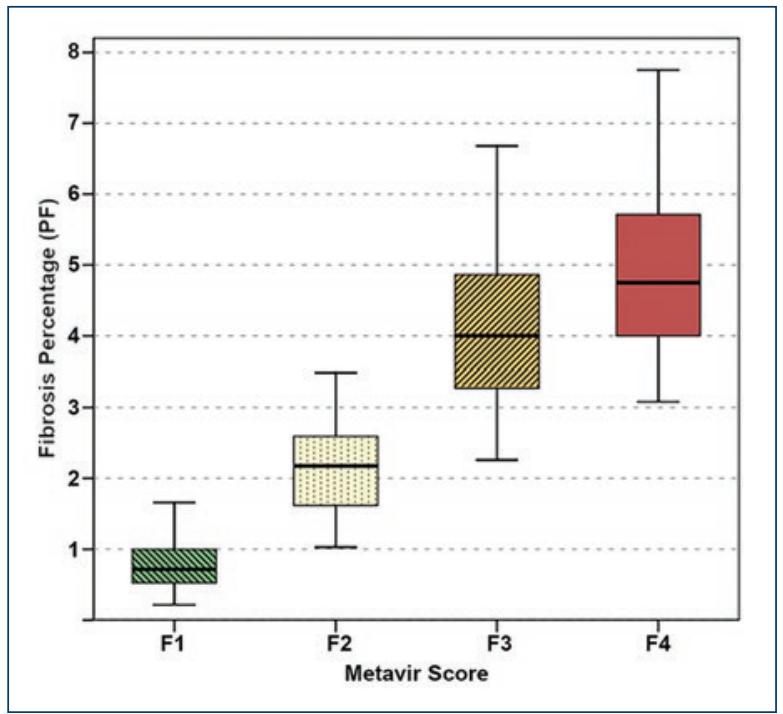

Figure 3. Box plot for the fibrosis percentage according to the evaluation groups.

evaluation groups ( $p=0.0001)$. To specify which groups differ from each other, a post hoc analysis was carried out and the statistical differences were found 
Table 4. Results of the Metavir evaluation and the percentage of fibrosis according to the induction groups

\begin{tabular}{|c|c|c|c|c|c|}
\hline \multirow[t]{2}{*}{ Induction group } & \multirow[t]{2}{*}{ Individual } & \multicolumn{2}{|c|}{ Metavir } & \multicolumn{2}{|c|}{ FP } \\
\hline & & Evaluation & Range & ${\overline{F P_{i}}}_{(\%)}$ & $S D_{F P_{i}(\%)}$ \\
\hline \multirow[t]{4}{*}{$\mathrm{T} 1$} & $A$ & $\mathrm{~F} 1$ & 1 & 0.8 & 0.5 \\
\hline & B & F3 & 2 & 2.8 & 0.8 \\
\hline & C & $\mathrm{F} 1$ & 1 & 1.4 & 0.3 \\
\hline & D & $\mathrm{F} 1$ & 2 & 0.9 & 0.9 \\
\hline \multirow[t]{4}{*}{ T2 } & A & F2 & 1 & 1.5 & 0.6 \\
\hline & B & F2 & 1 & 1.5 & 0.8 \\
\hline & C & $\mathrm{F} 1$ & 1 & 0.7 & 0.3 \\
\hline & D & F2 & 2 & 2.7 & 1.0 \\
\hline \multirow[t]{4}{*}{ T3 } & $A$ & F2 & 2 & 2.7 & 1.1 \\
\hline & B & F4 & 1 & 3.8 & 0.7 \\
\hline & C & F3 & 1 & 4.5 & 1.0 \\
\hline & D & F4 & 1 & 4.6 & 1.1 \\
\hline \multirow[t]{4}{*}{ T4 } & A & F4 & 0 & 5.6 & 1.5 \\
\hline & B & F4 & 0 & 5.1 & 0.9 \\
\hline & C & F3 & 1 & 4.3 & 1.2 \\
\hline & D & F4 & 1 & 5.2 & 1.0 \\
\hline
\end{tabular}

FP: fibrosis percentage.

to be significant in all cases $(p=0.0001)$. On the other hand, Spearman's rho test indicates a strong correlation between the percentage of fibrosis and histopathological diagnosis ( $p=0.0001, r=0.9)$.

\section{Discussion}

Among the alternatives of digital analysis for the images applied in the evaluation of hepatic fibrosis, it was found that some of the methods have important limitations, such as poor accuracy to detect perisinusoidal fibrosis and/or considerable coefficients of variation to evaluate cirrhosis. In most of the works, the image processing was done in the RGB color space; this feature mainly restricts the range of colors available. In addition, a full automation for the acquisition and processing of the images compromises the evaluation of defects and artifacts in tissue specimens. Many of the software were designed as a general purpose tool, and therefore, not specific for this pathology yielding important errors derived from the particularities in the hepatic architecture.
In this work, we present a morphometric method for the quantification of hepatic fibrosis, emphasizing its applicability as an auxiliary tool in the evaluation and diagnosis of this pathology. As shown in figure 1, most of the evaluated images in the induction Groups T1, T2, and $\mathrm{T} 4$ were in the expected phases $\mathrm{F} 1, \mathrm{~F} 2$, and $\mathrm{F} 4$, respectively. The dispersion of the liver fibrosis data suggested that the induction times did not generate a predominant stage of fibrosis but rather a dispersion of stages that are more evident in the T3 group in which $47.5 \%$ of the images evaluated corresponded to F4 and only $30 \%$ to $\mathrm{F} 3$ which was the expected phase. For the 160 images analyzed, there was a concordance of the three evaluators (3/3) in $70 \%$. However, 48 complex cases were also found, in which at least one of the observers did not coincide with the rest. It is also observed that for $\mathrm{F} 1$ the staging with a $3 / 3$ agreement was achieved in $58.5 \%$ of the cases, for $\mathrm{F} 2$ in $67.5 \%$, and for $\mathrm{F} 3$ in $66.7 \%$, while $\mathrm{F} 4$ had the highest number of cases totally concordant with $84.8 \%$. This analysis suggests that the inter-observer variability is greater in cases with incipient (F1) or moderate fibrosis (F2 and F3). 


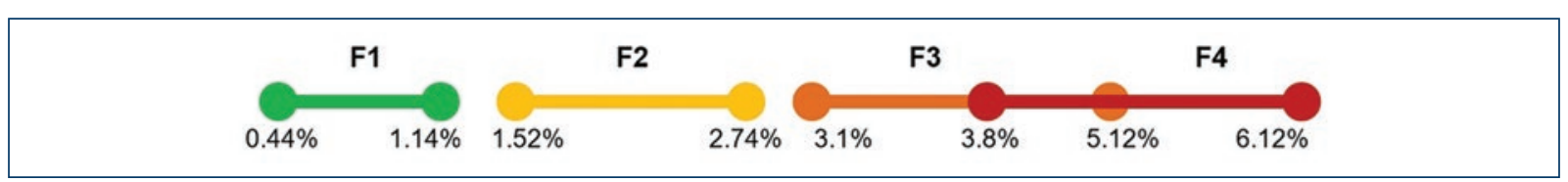

Figure 4. Fibrosis percentage score, statistically obtained for the fibrosis phases of the Metavir score.

The fibrosis evaluation was distributed as follows: the most populated group was the group with F4 evaluation, with $28.8 \%$ of the images, followed by $\mathrm{F} 1$ and $\mathrm{F} 2$ with $25.6 \%$ and $25 \%$, respectively. Group F3 is the least numerous representing $20.6 \%$ of cases. Despite having groups with a non-homogeneous population, it can be observed that the difference between the most populated and the least populated barely represents $8 \%$ of the total cases.

The results of the quantification of the FP range from $0.21 \%$ to $7.75 \%$. In figure 3 , it is possible to notice an increase in FP as the fibrosis phase is more advanced with a non-linear trend. A strong correlation was obtained between these two variables. Numerically, the average of the FP for each Metavir stage (Table 3) has an increase of $1.34 \%$ when going from $\mathrm{F} 1$ to $\mathrm{F} 2,1.98 \%$ in $\mathrm{F} 2$ to $\mathrm{F} 3$, and $0.85 \%$ in the transition from $\mathrm{F} 3$ to $\mathrm{F} 4$. Using this measurement and the SD for each group, FP ranges were calculated for each Metavir phase; this is illustrated in the diagram of figure 4 . The groups are distinguished from each other except for the overlap between Groups F3 and F4; however, the ANOVA test and its post hoc indicate that all the groups are statistically different from each other.

The method of induction of fibrosis by $\mathrm{CCl}_{4}$ was strict and carefully monitored to minimize errors; however, the animal model revealed a heterogeneous distribution of the disorder, within the same individual and between individuals of the same group corresponding to the same induction group: T1, T2, T3y, and T4. This is evidenced in the individual-by-individual analysis for each group, as shown in table 4, where, at least one individual was evaluated in a different Metavir stage than that expected, (this is to say), T1 was expected to correspond to F1 and so on to T4/F4. Especially, an ethereal behavior is observed in the individual $B$ of the Group T1, which evolved to F3 phase, even when it was submitted only to 4 weeks of induction. The T3 group was the most seriously affected since only one out of four individuals was evaluated in expected phase, F3.

The dispersion of fibrosis within the same individual can be seen through the Metavir and FP evaluation. For the first case, $12.5 \%$ of the individuals showed a rank equal to 0 , that is, only in two of the 16 rats, all the observations were evaluated in the same Metavir phase. On the other hand, $25 \%$ of the individuals of the model have a rank in Metavir evaluations equal to two that means that in four rats, up to three different phases were found in the same individual. Regarding the FP, it was observed that the SD, of the quantification per individual goes from $0.3 \%$ to $1.5 \%$. The average SD of the individuals staged in $\mathrm{F} 1$ is equal to $0.5 \%, 0.86 \%$ for $\mathrm{F} 2$, $1 \%$ for $F 3$, and $1.0 \%$ for $F 4$. The evaluation of these two methodologies suggests that the use of the Metavir score, yield more robust diagnoses for F4, since observations are based on shape characteristics, namely, the observation of complete nodules, regardless of the amount of fibrosis, or the thickness of the septa that form those nodules. This same discussion could justify the dispersion of FP that was observed in Group T4 and that is greater ( $>0.9$ for all individuals) than in the other induction groups.

A qualitative evaluation such as anatomo-histopathological assessment does not allow complete precision in the appraisal of liver damage once liver cirrhosis has been diagnosed. A more precise quantification of fibrosis would facilitate a distinction in the staging of liver cirrhosis, where a quantitative measurement is fundamental to evaluate the effect of treatments for the control or reversion of the pathology. Therefore, the quantification of FP by digital morphometry can increase the resolution for a complete evaluation of liver fibrosis, especially in intermediate stages.

\section{Conclusions}

In conclusion, this study confirms the use of HepaScan as a quantitative tool to evaluate the amount of fibrosis in liver biopsy images in a murine model of hepatic fibrosis. The morphometric quantification in terms of the fibrosis percentage FP had a high correlation with the histopathological evaluation of images of histological sections of liver biopsies. The quantification of the FP by digital morphometry will potentiate its use in the evaluation of the effect of treatments for the control or reversion of the pathology. 


\section{Conflicts of interest}

The authors declare that they have no conflicts of interests.

\section{Funding}

This project received partial financial support by DGAPA-UNAM- PAPIIT through grants IT100518.

\section{Ethical disclosures}

Protection of human and animal subjects. The authors declare that the procedures followed were in accordance with the regulations of the relevant clinical research ethics committee and with those of the Code of Ethics of the World Medical Association (Declaration of Helsinki).

Confidentiality of data. The authors declare that no patient data appear in this article.

Right to privacy and informed consent. The authors declare that no patient data appear in this article.

\section{References}

1. Méndez-Sánchez N,Uribe M. Hepatología. Conceptos básicos y clínicos Mc Graw-Hill. Mexico City, Mexico, 2016.

2. Mokdad AA, Lopez AD, Shahraz S, Lozano R, Mokdad AH, Stanaway J, et al. Liver cirrhosis mortality in 187 countries between 1980 and 2010 : a systematic analysis. BMC Med. 2014;12:145.

3. Diagnóstico General de la Salud Poblacional. Subsecretaría de Integración y Desarrollo del Sector Salud, Dirección General de Evaluación del
Desempeño. México: Diagnóstico General de la Salud Poblacional; 2015. p. 108-10.

4. Bustíos C, Dávalos M, Román R, Zumaeta E. Características epidemiológicas y clínicas de la cirrosis hepática en la unidad de hígado del hnerm es-salud. Rev Gastroenterol Perú. 2007;27:238-45.

5. Hernandez-Gea V, Friedman SL. Pathogenesis of liver fibrosis. Annu Rev Pathol. 2011;6:425-56.

6. Arroyo J, Almora Y, Quino M, Raez E, Martínez J, Buendía J, et al. Efecto protector en cirrosis hepática inducida en ratas del extracto etanólico de las hojas de Piper aduncum comparado con silimarina. An Fac Med. 2012;73:85-91.

7. Herrera A, González M, Céspedes E, Sánchez SG. Efectos del alcoholismo crónico sobre el hígado de ratas albinas adolescentes. Rev Cubana Invest Bioméd. 1999;18:189-96.

8. Atucha N, Ramirez M, Pérez M, Martínez C, Sánchez A, Quesada T, et al. Efectos de la inhibición de la síntesis de óxido nítrico en la hemodinámica y excreción renal de ratas con cirrosis biliar crónica. Nefrología. 1994; 14:656-62.

9. Regev A, Berho M, Jeffers LJ, Milikowski C, Molina EG, Pyrsopoulos NT, et al. Sampling error and intraobserver variation in liver biopsy in patients with chronic HCV infection. Am J Gastroenterol. 2002:97:2614-8.

10. Ausina V, Moreno S. Tratado SEIMC de Enfermedades Infecciosas y Microbiología Clínica. Argentina: Médica Panamericana; 2005. p. 977-82.

11. Dahab GM, Kheriza MM, El-Beltagi HM, Fouda AM, El-Din OA. Digital quantification of fibrosis in liver biopsy sections: description of a new method by Photoshop software. J Gastroenterol Hepatol. 2004; 19:78-85.

12. Goodman ZD, Becker RL Jr., Pockros PJ, Afdhal NH. Progression of fibrosis in advanced chronic hepatitis C: evaluation by morphometric image analysis. Hepatology. 2007;45:886-94.

13. Zaitoun AM, Al Mardini H, Awad S, Ukabam S, Makadisi S, Record CO. Quantitative assessment of fibrosis and steatosis in liver biopsies from patients with chronic hepatitis C. J Clin Pathol. 2001;54:461-5.

14. Lin XZ, Horng MH, Sun YN, Shiesh SC, Chow NH, Guo XZ. Computer morphometry for quantitative measurement of liver fibrosis: comparison with Knodell's score, colorimetry and conventional description reports. J Gastroenterol Hepatol. 1998:13:75-80.

15. Lazzarini AL, Levine RA, Ploutz-Snyder RJ, Sanderson SO. Advances in digital quantification technique enhance discrimination between mild and advanced liver fibrosis in chronic hepatitis C. Liver Int. 2005; 25:1142-9.

16. Manibusan MK, Odin M, Eastmond DA. Postulated carbon tetrachloride mode of action: a review. J Environ Sci Health C Environ Carcinog Ecotoxicol Rev. 2007;25:185-209.

17. Jang JH, Kang KJ, Kim YH, Kang YN, Lee IS. Reevaluation of experimental model of hepatic fibrosis induced by hepatotoxic drugs: an easy, applicable, and reproducible model. Transplant Proc. 2008; 40:2700-3. 